\title{
A CENTRAL LIMIT THEOREM ON HEISENBERG TYPE GROUPS. II
}

\author{
PETER OHRING
}

(Communicated by Palle E. T. Jorgensen)

\begin{abstract}
We present a Liapounov type central limit theorem for random variables associated to a commutative Banach algebra of "radial" measures on Heisenberg type groups. This theorem improves on a result presented by the author in Proc. Amer. Math. Soc. 113 (1991), 529-536.
\end{abstract}

\section{INTRODUCTION}

In [Oh] we introduced a commutative algebra of "radial", bounded, Borel measures on Heisenberg type groups (H-type groups). For probability measures in this algebra satisfying certain integrability conditions we proved a central limit theorem analogous to one of the classical Euclidean versions [Oh, Theorem 4.1]). The proof exploited explicit formulas for the Gelfand transform on the above mentioned commutative algebra.

In this paper we present a Liapounov type version of the central limit theorem on H-type groups. The awkward integrability hypothesis of [Oh] is replaced by a more standard third moment integrability hypothesis.

As in [Oh] we exploit some of the many parallels between analysis on $\mathrm{H}$ type groups and Euclidean analysis (cf. [Fa]). In particular use is made of homogeneous Taylor polynomials on H-type groups.

The main result is presented in $\S 2$. Section 1 is devoted to preliminaries on $\mathrm{H}$ type groups, homogeneous Taylor polynomials, group valued random variables, and the heat semigroup that provides us with a notion of normal distributions.

\section{Preliminaries}

A group of type $\mathrm{H}$ is a connected, simply connected, real Lie group whose Lie algebra is of type $H$. A Lie algebra $n$ is of type $H$ if $n=v \oplus z ; \mathfrak{v}, \mathfrak{z}$ real Euclidean spaces, with a Lie algebra structure such that $z$ is the center of $n$ and for all $v \in \mathfrak{v}$ of length one, $a d_{v}$ is a surjective isometry of the orthogonal complement of ker $a d_{v}$ in $\mathfrak{v}$, onto $\mathfrak{z}$.

Let $\mathcal{N}$ be a type $\mathrm{H}$ group and $\mathfrak{n}=\mathfrak{v} \oplus \mathfrak{z}$ its Lie algebra. There is a natural dilation structure on $\mathscr{N}$. For $s>0$ define $\delta_{s}(v, z)=\left(s v, s^{2} z\right)$.

Received by the editors August 20, 1991 and, in revised form, November 30, 1991.

1991 Mathematics Subject Classification. 22E27, 60B15; Secondary 43A80. 
We fix a basis $X_{1}, X_{2}, \ldots, X_{n}$ for $\mathfrak{n}$ consisting of eigenvectors for the dilations $\delta_{s}$ with eigenvalues $r^{d_{1}}, \ldots, r^{d_{n}} \quad\left(d_{i}=1\right.$ or 2$)$ in such a way that $X_{1}, X_{2}, \ldots, X_{\operatorname{dim} \mathfrak{v}}$ forms a basis for $\mathfrak{v}$. For a multi-index $I=\left(i_{1}, i_{2}, \ldots, i_{n}\right)$ let $d(I)=d_{1} i_{1}+d_{2} i_{2}+\cdots+d_{n} i_{n} . d(I)$ is the homogeneous degree of $X^{I}=$ $X_{1}^{i_{1}} \cdots X_{n}^{i_{n}}$.

The left Taylor polynomial of $f$ at $g$ of homogeneous degree $a$ is the unique homogeneous polynomial $P$ of homogeneous degree less than or equal to $a$ such that $X^{I} P(0)=X^{I} f(g)$ for all multi-indices $I$ with $d(I) \leq a$.

In [FS, Theorem 1.42] Folland and Stein prove that if $f \in C^{k+1}(\mathcal{N})$ with bounded derivatives of order $(k+1)$ and $P_{g}$ is the left Taylor polynomial of homogeneous degree $k$, then

$$
\left|f\left(g g^{\prime}\right)-P_{g}\left(g^{\prime}\right)\right| \leq K|g|^{k+1} \quad \text { for } g, g^{\prime} \in \mathcal{N} .
$$

(Here $|g|$ denotes the homogeneous norm of $g$ on $\mathscr{N}$.)

For example, on the three-dimensional Heisenberg whose Lie algebra is spanned by three vectors $X, Y, Z,[X, Y]=Z$, the left Taylor polynomial of $f$ at $g$ of homogeneous degree 2 is given by

$$
\begin{aligned}
P_{g}(x, y, z)= & f(g)+(X f)(g) x+(Y f)(g) y+(Z f)(g) z \\
& +\frac{\left(X^{2} f\right)(g)}{2 !} x^{2}+\frac{\left(Y^{2} f\right)(g)}{2 !} y^{2}+\frac{((X Y+Y X) f)(g)}{2 !} x y
\end{aligned}
$$

where $g \in \mathscr{N}$. Thus it follows that

$$
\left|f\left(g g^{\prime}\right)-P_{g}\left(g^{\prime}\right)\right| \leq K\left|g^{\prime}\right|^{3} \quad \text { for } g, g^{\prime} \in \mathcal{N},
$$

provided $f$ has uniformly bounded third derivatives.

An $\mathscr{N}$-valued random variable is a measurable function from some probability space $(\Omega, \mathscr{F}, \mathscr{P})$ to $\mathscr{N}$. For each $\mathscr{N}$ random variable $\xi$ we can define a probability measure $\mu_{\xi}$ on $\mathscr{N}$ by $\mu_{\xi}(A)=\mathscr{P}\left(\xi^{-1}(A)\right), A \subset \mathscr{N}$.

If $\varphi: \mathscr{N} \rightarrow \mathbf{R}$ we define the $\varphi$ expectation of the random variable $\xi$ to be

$$
\varepsilon_{\varphi}(\xi)=\int_{\mathscr{N}} \varphi(g) d \mu_{\xi}(g)
$$

For $F: \mathscr{N} \rightarrow \mathscr{N}$ we can define a random variable $F(\xi)$ by composition. The $\varphi$ expectation of this random variable is given by

$$
\varepsilon_{\varphi}(F(\xi))=\int_{\mathscr{N}} \varphi(F(g)) d \mu_{\xi}(g) .
$$

If $\varphi$ is one of the coordinate functions, $\varphi\left(x_{1}, x_{2}, \ldots, x_{n}\right)=x_{i}$ for some $0 \leq i \leq n$, then $\varepsilon_{x_{i}}$ will denote the corresponding expectation.

A measurable function $\alpha: \Omega \times \Omega \rightarrow \mathscr{N} \times \mathscr{N}$ is a vector valued $\mathscr{N}$-random variable. Let $\mu_{\alpha}^{2}$ be the corresponding probability measure on $\mathscr{N} \times \mathscr{N}$. The prime example of this that we will use is $\alpha\left(\omega_{1}, \omega_{2}\right)=\left(\xi\left(\omega_{1}\right), \eta\left(\omega_{2}\right)\right)$ for two independent random variables $\xi$ and $\eta$, in which case $\mu_{\alpha}^{2}$ equals the product of the measures $\mu_{\xi}$ and $\mu_{\eta}$.

Let $F: \mathscr{N} \times \mathscr{N} \rightarrow \mathscr{N}$. Then

$$
\varepsilon_{\varphi}(F(\alpha))=\iint_{\mathscr{N} \times \mathscr{N}} \varphi\left(F\left(g, g^{\prime}\right)\right) d \mu_{\xi} d \mu_{\eta} .
$$


For $F\left(g, g^{\prime}\right)=g \cdot g^{\prime}$ we have

$$
\varepsilon_{\varphi}(F(\alpha))=\iint_{\mathscr{N} \times \mathscr{N}} \varphi\left(g \cdot g^{\prime}\right) d \mu_{\xi} d \mu_{\eta} .
$$

This generalizes in a straightforward manner to products of more than two independent random variables. (For more on group-valued random variables see $[\mathrm{He}]$.)

A bounded measure $\mu \in M_{b}(\mathscr{N})$ is said to be $\mathfrak{v}$ radial if $d \mu(A v, z)=$ $d \mu(v, z)$ for all $A \in O(\mathfrak{v})$, the orthogonal group of $\mathfrak{v}$. Let $M_{b}(\mathcal{N})^{\sharp}$ denote the Banach algebra generated by the $\mathfrak{v}$-radial measures. In [Oh] we showed that $M_{b}(\mathscr{N})^{\sharp}$ is a commutative Banach algebra.

Let $\Delta$ be the usual Laplacian on $\mathfrak{v}$. We will denote by $\left\{p_{t}\right\}_{t>0}$ the semigroup of solutions of the heat equation corresponding to $\Delta$ on the group $\mathcal{N}$. The following properties of $p_{t}$ are well known (cf. [Hu]):

(1) $p_{t}>0$.

(2) $p_{t}$ is $\mathfrak{v}$-radial and in $C^{\infty}(\mathcal{N})$.

(3) $\iint p_{t}(v, z) d v d z=1$.

If $t \cdot(v, z)$ denotes dilation of $(v, z)$ by $t$ then it is well known that the heat semigroup satisfies

$$
p_{t}(v, z)=t^{-Q / 2} p_{1}\left(t^{-1 / 2} \cdot(v, z)\right),
$$

where $Q=\operatorname{dim} \mathfrak{v}+2 \operatorname{dim} \mathfrak{z}$ is the homogeneous dimension of the group. Furthermore it is well known that the heat semigroup is rapidly decaying at infinity. It follows that

$$
\begin{aligned}
& \int_{\mathscr{N}}|(v, z)|^{3} p_{t}(v, z) d v d z=\int_{\mathscr{N}}|(v, z)|^{3} t^{-Q / 2} p_{1}\left(t^{-1 / 2} \cdot(v, z)\right) d v d z \\
& \quad=\int_{\mathscr{N}}\left|t^{1 / 2} \cdot(v, z)\right|^{3} p_{1}(v, z) d v d z=t^{3 / 2} \int_{\mathscr{N}}|(v, z)|^{3} p_{1}(v, z) d v d z .
\end{aligned}
$$

A similar calculation shows that

$$
\int_{\mathscr{N}}|v|^{2} p_{t}(v, z) d v d z=t \int_{\mathscr{N}}|v|^{2} p_{1}(v, z) d v d z .
$$

Equations (1.1) and (1.2) imply that

$$
\int_{\mathscr{N}}|(v, z)|^{3} p_{t}((v, z)) d v d z=c\left(\int_{\mathscr{N}}|v|^{2} p_{t}(v, z) d v d z\right)^{3 / 2}
$$

for some constant $c$. We will exploit this relationship in the sequel.

\section{MAIN Result}

In this section we present our central limit theorem. The statement and proof are based on Liapounov's and Lindeberg's theorem and proof, respectively (cf. [Ch]). We follow the notation used in [Ch] whenever possible. In the sequel expressions of the form $\xi / s, \xi$ an $\mathscr{N}$-valued random variable and $s$ a positive real number should be interpreted as the $\mathscr{N}$-valued random variable given by composition of $\xi$ with $\delta_{s^{-1}}$. 
Theorem 2.1. Let $\left\{\xi_{j}\right\}$ be a sequence of independent $\mathscr{N}$-valued random variables such that

(1) $\mu_{\xi_{j}} \in M_{b}(\mathscr{N})^{\sharp}$;

(2) $\varepsilon_{\varphi}\left(\xi_{j}\right)=0$ for all $\varphi$ of the form $\varphi(v, z)=\left\langle z, z^{\prime}\right\rangle, z^{\prime} \in \mathfrak{z}$;

(3) $\sigma^{2}\left(\xi_{j}\right)=\sigma_{j}^{2}=\varepsilon_{\varphi}\left(\xi_{j}\right)<\infty$, where $\varphi(v, z)=|v|^{2}$;

(4) $\varepsilon\left(\left|\xi_{j}\right|^{3}\right)=\gamma_{j}=\varepsilon_{\varphi}\left(\xi_{j}\right)<\infty$, where $\varphi(v, z)=|(v, z)|^{3}$.

Set

$$
S_{m}=\prod_{j=1}^{m} \xi_{j}, \quad s_{m}^{2}=\sum_{j=1}^{m} \sigma_{j}^{2}, \quad \Gamma_{m}=\sum_{j=1}^{m} \gamma_{j} .
$$

If $\Gamma_{m} / s_{m}^{3} \longrightarrow 0$ as $m \longrightarrow \infty$ then $\mu_{S_{m} / s_{m}} \longrightarrow p_{1} d v d z$ weakly, where $p_{1}$ is the element of the heat semigroup corresponding to $t=1$.

Proof. The idea is to approximate $\xi_{1} \cdot \xi_{2} \cdots \xi_{m}$ by replacing one $\xi$ at a time with a comparable "normal" random variable $\zeta$ as follows: Let $\left\{\zeta_{j}\right\}_{j=1}^{\infty}$ be $\mathscr{N}$-valued random variables having absolutely continuous distributions with Radon-Nikodym derivatives $p_{\sigma_{j}}$ (from heat semigroup). Let all the $\xi_{j}$ 's and $\zeta_{j}$ 's be totally independent. Set

$$
\eta_{j}=\zeta_{1} \cdots \zeta_{j-1} \cdot \xi_{j+1} \cdots \xi_{m}, \quad 1 \leq j \leq m,
$$

with the convention that

$$
\eta_{1}=\xi_{2} \cdots \xi_{m}, \quad \eta_{m}=\zeta_{1} \cdots \zeta_{m-1} .
$$

Let $f: \mathscr{N} \rightarrow \mathscr{N}$ be $C^{3}$ with bounded derivatives of orders up to and including three. Since all the measures $\mu_{\xi_{j}}, \mu_{\eta_{j}}$, and $\mu_{\zeta_{j}}$ commute, we have

$$
\begin{aligned}
\varepsilon_{x_{i}}\left\{f\left(\frac{\xi_{1} \cdots \xi_{m}}{s_{m}}\right)\right\}-\varepsilon_{x_{i}}\left\{f\left(\frac{\zeta_{1} \cdots \zeta_{m}}{s_{m}}\right)\right\} \\
=\sum_{j=1}^{m}\left[\varepsilon_{x_{i}}\left\{f\left(\frac{\xi_{j} \eta_{j}}{s_{m}}\right)\right\}-\varepsilon_{x_{i}}\left\{f\left(\frac{\zeta_{j} \eta_{j}}{s_{m}}\right)\right\}\right],
\end{aligned}
$$

for all $1 \leq i \leq n$.

We would like to estimate the terms in the right-hand side of $(2.1)$. Let $f^{1}, \ldots, f^{n}$ be the components of $f$ and $P_{g}^{1}, \ldots, P_{g}^{n}$ the corresponding homogeneous Taylor polynomials of degree 2 at $g$. It follows from the definition of the expectation of an $\mathscr{N}$-valued random variable and the Taylor polynomial that

$$
\begin{aligned}
& \left|\varepsilon_{x_{i}}\{f(\xi \eta)\}-\varepsilon_{x_{i}}\left\{P_{\eta}(\xi)\right\}\right|=\left|\iint_{\mathcal{N} \times \mathscr{N}}\left(f^{i}\left(g g^{\prime}\right)-P_{g}^{i}\left(g^{\prime}\right)\right) d \mu_{\xi} d \mu_{\eta}\right| \\
& \quad \leq \iint_{\mathscr{N} \times \mathscr{N}}\left|f^{i}\left(g g^{\prime}\right)-P_{g}^{i}\left(g^{\prime}\right)\right| d \mu_{\xi} d \mu_{\eta} \leq M \int_{\mathcal{N}}\left|g^{\prime}\right|^{3} d \mu_{\xi}=M \varepsilon\left\{|\xi|^{3}\right\}
\end{aligned}
$$

where $M$ represents a constant that depends on $f$ and $i$. A similar argument can be carried out with $\zeta$ replacing $\xi$. Putting this all together we obtain

$$
\left|\varepsilon_{x_{i}}\{f(\xi \eta)\}-\varepsilon_{x_{i}}\{f(\zeta \eta)\}+\varepsilon_{x_{i}}\left\{P_{\eta}(\zeta)\right\}-\varepsilon_{x_{i}}\left\{P_{\eta}(\xi)\right\}\right| \leq M \varepsilon\left\{|\xi|^{3}+|\zeta|^{3}\right\} .
$$

Our choice of $\zeta_{j}$ implies $\varepsilon_{x_{i}}\left\{P_{\eta_{j}}\left(\zeta_{j}\right)\right\}=\varepsilon_{x_{i}}\left\{P_{\eta_{j}}\left(\xi_{j}\right)\right\}$ : To see this we note that

$$
P_{g}^{i}\left(g^{\prime}\right)=f^{i}(g)+\sum_{k=1}^{n}\left(X_{k} f^{i}\right)(g) x_{k}+\frac{1}{2} \sum_{k, l}\left(X_{k} X_{l} f^{i}\right)(g) x_{j} x_{k},
$$


where $g^{\prime}=\left(x_{1}, x_{2}, \ldots, x_{n}\right)$, and where the last sum is over $k, l=1,2, \ldots$, $\operatorname{dim} \mathfrak{v}$. It follows that

$$
\begin{aligned}
\varepsilon_{x_{i}}\left\{P_{\eta_{j}}\left(\xi_{j}\right)\right\}= & \iint_{\mathscr{N} \times \mathscr{N}} P_{g}^{i}\left(g^{\prime}\right) d \mu_{\xi_{j}}\left(g^{\prime}\right) d \mu_{\eta_{j}}(g) \\
= & \int_{\mathscr{N}} f^{i}(g) d \mu_{\eta_{j}}(g)+\sum_{k=1}^{n}\left(\int_{\mathscr{N}}\left(X_{k} f^{i}\right)(g) d \mu_{\eta_{j}}(g) \cdot \int_{\mathscr{N}} x_{k} d \mu_{\xi_{j}}\left(g^{\prime}\right)\right) \\
& +\frac{1}{2} \sum_{k, l}\left(\int_{\mathscr{N}}\left(X_{k} X_{l} f^{i}\right)(g) d \mu_{\eta_{j}}(g) \cdot \int_{\mathscr{N}} x_{k} x_{l} d \mu_{\xi_{j}}\left(g^{\prime}\right)\right)
\end{aligned}
$$

The terms in the first sum are equal to zero as a consequence of hypotheses (1) and (2) of Theorem 2.1. Terms in the second sum are equal to zero when $k \neq l: x_{k} x_{l}$ is integrable with respect to $\mu_{\xi}$ as a consequence of hypothesis (3) in Theorem 2.1 and the simple observation that $\left|x_{k} x_{l}\right| \leq \frac{1}{2}\left(x_{k}^{2}+x_{l}^{2}\right)$. Since $\mu_{\xi}$ is $\mathfrak{v}$-radial, a rotation of $\pi$ radians in the $x_{k} x_{l}$-plane yields

$$
\int_{\mathscr{N}} x_{k} x_{l} d \mu_{\xi_{j}}\left(g^{\prime}\right)=-\int_{\mathscr{N}} x_{l} x_{k} d \mu_{\xi_{j}}\left(g^{\prime}\right) \text {. }
$$

Hence

$$
\varepsilon_{x_{i}}\left\{P_{\eta_{j}}\left(\xi_{j}\right)\right\}=\int_{\mathcal{N}} f^{i}(g) d \mu_{\eta_{j}}(g)+\frac{1}{2} \operatorname{dim} \mathfrak{v} \cdot \sigma_{j}^{2} \cdot \sum_{k=1}^{\operatorname{dim} \mathfrak{v}} \int_{\mathscr{N}}\left(X_{k}^{2} f^{i}\right)(g) d \mu_{\eta_{j}}(g) .
$$

Similar considerations lead to the the same value for $\varepsilon_{x_{i}}\left\{P_{\eta_{j}}\left(\zeta_{j}\right)\right\}$.

Using this, the inequality in (2.2) becomes

$$
\left|\varepsilon_{x_{i}}\{f(\xi \eta)\}-\varepsilon_{x_{i}}\{f(\zeta \eta)\}\right| \leq M \varepsilon\left\{|\xi|^{3}+|\zeta|^{3}\right\}
$$

Substituting $\xi_{j} / s_{m}, \eta_{j} / s_{m}, \zeta_{j} / s_{m}$ for $\xi, \eta, \zeta$ into this inequality and returning to $(2.1)$, we obtain

$$
\begin{aligned}
& \left|\varepsilon_{x_{i}}\left\{f\left(\frac{\xi_{1} \cdots \xi_{m}}{s_{m}}\right)\right\}-\varepsilon_{x_{i}}\left\{f\left(\frac{\zeta_{1} \cdots \zeta_{m}}{s_{m}}\right)\right\}\right| \\
& \quad \leq M \sum_{j=1}^{m}\left\{\frac{\varepsilon\left\{\left|\xi_{j}\right|^{3}\right\}}{s_{m}^{3}}+\frac{\varepsilon\left\{\left|\zeta_{j}\right|^{3}\right\}}{s_{m}^{3}}\right\}=M \sum_{j=1}^{m}\left\{\frac{\gamma_{j}}{s_{m}^{3}}+\frac{\varepsilon\left\{\left|\zeta_{j}\right|^{3}\right\}}{s_{m}^{3}}\right\} \\
& \quad \leq M^{\prime} \sum_{j=1}^{m} \frac{\gamma_{j}}{s_{m}^{3}}=M^{\prime} \frac{\Gamma_{m}}{s_{m}^{3}} .
\end{aligned}
$$

In this last inequality we have used the fact that $\sigma_{j}^{3} \leq \gamma_{j}$ (Hölder's inequality with $p=3 / 2$ ) and that $\varepsilon\left\{\left|\zeta_{j}\right|^{3}\right\}=c \sigma_{j}^{3}$ for some constant $c$ (follows directly from the relationship in (1.3) and the definitions of these expectations).

Thus we have shown that for all $f: \mathcal{N} \rightarrow \mathscr{N}$ with bounded derivatives of orders up to and including three, and for all $i=1,2, \ldots, n$,

$$
\left|\varepsilon_{x_{i}}\left\{f\left(\frac{S_{m}}{s_{m}}\right)\right\}-\varepsilon_{x_{i}}\{f(N)\}\right| \leq O\left(\frac{\Gamma_{m}}{s_{m}^{3}}\right)
$$

where $N$ denotes a random variable with probability distribution $p_{1}$. These functions are dense $C_{0}(\mathscr{N})$. The last inequality is equivalent to

$$
\left|\int_{\mathscr{N}} f^{i}(g) d\left(\mu_{\xi_{1} / s_{m}} * \mu_{\xi_{2} / s_{m}} * \cdots * \mu_{\xi_{m} / s_{m}}\right)-\int_{\mathscr{N}} f^{i}(g) p_{1}(g) d g\right| \leq O\left(\frac{\Gamma_{m}}{s_{m}^{3}}\right)
$$


since by the definition of convolution

$$
\int_{\mathscr{N} \cdots \mathscr{N}} \cdots \int^{i}\left(g_{1} \cdots g_{m}\right) d \mu_{\xi_{1} / s_{m}} \cdots d \mu_{\xi_{n} / s_{m}}=\int_{\mathscr{N}} f^{i}(g) d\left(\mu_{\xi_{1} / s_{m}} * \cdots * \mu_{\xi_{m} / s_{m}}\right) .
$$

Thus we obtain the required weak convergence.

We would like to thank Michael Lacey for suggesting this approach to the central limit theorem.

\section{REFERENCES}

[Ch] K. L. Chung, A course in probability theory, Academic Press, New York, 1974.

[Fa] J. Faraut and K. Harzallah, Deux cours d'analyse harmonique, Birkhäuser, Basel, 1987.

[F-S] G. B. Folland and E. M. Stein, Hardy spaces on homogeneous groups, Princeton Univ. Press, Princeton, NJ, 1982.

[He] H. Heyer, Probability measures on locally compact groups, Springer-Verlag, Berlin and New York, 1977.

[Hu] A. Hulanicki, A class of convolution semi-groups of measures on a Lie group, Lecture Notes in Math., vol. 828, Springer-Verlag, Berlin and New York, 1980, pp. 82-101.

[Oh] P. Ohring, A central limit theorem on Heisenberg type groups, Proc. Amer. Math. Soc. 113 (1991), 529-536.

Department of Mathematics, State University of New York at Purchase, 735 AnderSon Hill Road, Purchase, New York 10577-1400 